\title{
LE DESC D'ANDROLOGIE
}

\section{Année universitaire 1997-1998}

Responsable: $\quad$ Pr J.C. CZYBA

Biologie de la Reproduction

Faculté de Médecine Grange Blanche

8 , Avenue Rockefeller

69373 Lyon Cedex 08

Tél. : 04-78-77-70-65 ; Fax : 04-78-01-49-75

Formalités d'inscription : Mme Claire RACHEL 3ème cycle «spécialités»

Domaine Rockefeller

8, Avenue Rockefeller 69373 Lyon Cedex 08

Tél. : 04-78-77-70-87 ; Fax : 04-78-77-71-58

Commissions inter-régionales :

ILE DE FRANCE : Coordonnateur :

Pr. JOUANNET : PARIS

Membres :

Pr. JARDIN (PARIS)

Pr. BOUCHARD (PARIS)

Pr. THIBAULT (PARIS)

RHONE-ALPES Coordonnateur : Pr. PUGEAT : LYON

Membres :

Pr. CZYBA (LYON)

Pr. LERICHE (LYON)

Pr. RAMBEAUD (GRENOBLE)

NORD-EST

Coordonnateur :

Pr. JACQMIN : STRASBOURG

Membres :

Pr. FOLLIGUET (NANCY)

Pr. LECLERE (NANCY)

Pr. CLAVERT (STRASBOURG)

NORD-OUEST Coordonnateur : Pr. KUHN : ROUEN

Membres :

Pr. THEPOT (AMIENS)

Pr. PETIT (AMIENS)

Pr. BENSADOUN (CAEN)

OUEST

Coordonnateur :

Pr. LOBEL : RENNES

Membres :

Pr. CHARBONNEL (NANTES)

Pr. ROYERE (TOURS)

Pr. LELANNOU (RENNES) 
SUD

Coordonnateur:

Pr. NAVRATIL : MONTPELLIER

Membres :

Pr. BRINGER (MONTPELLIER)

Pr. BOUCHER (CLERMONT-FERRAND)

Pr. ROSSI (MARSEILLE)

SUD-OUEST

Coordonnateur :

Pr. PONTONNIER : TOULOUSE

Membres :

Pr. LEGUILLOU (BORDEAUX)

Pr. MAYER (BORDEAUX)

Pr. LOUVET (TOULOUSE)

\section{PROGRAMME}

L'enseignement théorique sera dispensé à Lyon, en 1998 :

du Lundi 2 Mars au Vendredi 6 Mars

du Lundi 8 Juin au Vendredi 12 Juin.

Salle de Réunion du Laboratoire de Biologie de la Reproduction, Escalier A, étage V Faculté de Médecine 8, Avenue Rockefeller 69008 Lyon

\section{1ère Semaine}

Lundi 2 Mars

$10 \mathrm{~h}$ à $12 \mathrm{~h}$

Pr. CZYBA

Ontogenèse de la sexualité

$14 \mathrm{~h}$ à $16 \mathrm{~h}$

Dr. DUBANCHET

Développement psychosexuel

$16 \mathrm{~h}$ à $18 \mathrm{~h}$

Pr. PUGEAT

Hormones et sexualité

$18 \mathrm{~h}$ à $19 \mathrm{~h}$

Dr. ROLLET

La libido et ses perturbations

Mardi 3 Mars

$9 \mathrm{~h}$ à $12 \mathrm{~h}$

Dr. GIULIANO

Anatomo-physiologie de l'érection, de l'éjaculation et de l'orgasme

$14 \mathrm{~h}$ à $16 \mathrm{~h}$

Mr. BEJIN

L'orgasme au masculin

$16 \mathrm{~h}$ à $18 \mathrm{~h}$

Dr. CHEVRET et

Interrogatoire et évaluation d'un trouble sexuel chez l'homme

Pr. LERICHE

Mercredi 4 Mars

$9 \mathrm{~h}$ à $11 \mathrm{~h}$

$11 \mathrm{~h}$ à $12 \mathrm{~h}$ et

$14 \mathrm{~h}$ à $16 \mathrm{~h}$

$16 \mathrm{~h}$ à $18 \mathrm{~h}$
Dr. BONDIL

Pr. JARDIN

Dr. DUBANCHET
Méthodes d'exploration de l'érection

Dysfonctions sexuelles organiques et iatrogènes

Névrose et Sexualité. Perversions sexuelles
Jeudi 5 Mars

$9 \mathrm{~h}$ à $11 \mathrm{~h}$

$11 \mathrm{~h}$ à $12 \mathrm{~h}$

$14 \mathrm{~h}$ à $16 \mathrm{~h}$

$16 \mathrm{~h}$ à $18 \mathrm{~h}$
MmeBERNARDPIOOD

Dr.RIBES

Pr.NAVRATIL

Pr.LERICHE

Vendredi 6 Mars

$9 \mathrm{~h}$ à $12 \mathrm{~h}$ Dr.CHEVRET
Sexualité de l'enfant et de l'adolescent. Information sexuelle Sexualité et vieillissement Courbures de la verge et maladie de La Peyronie Micropénis. Allongement de la verge. Priapisme

Dysfonctions sexuelles psychologiques. La femme de l'impuissant et de l'éjaculateur précoce 
$14 \mathrm{~h}$ à $16 \mathrm{~h}$

$16 \mathrm{~h}$ à $18 \mathrm{~h}$

Dr. ROCHET

Pr. FAURE

$18 \mathrm{~h}$ à $19 \mathrm{~h}$
MST, Sida, Toxicomanies et Sexualité

Affections dermato-vénérologiques de la verge

Conduite à tenir devant une ambiguiité sexuelle à la naissance

\section{2ème Semaine}

Lundi 8 Juin

$10 \mathrm{~h}$ à $12 \mathrm{~h}$

Dr. CHEVRET

Introduction aux différents modes de thérapie

$14 \mathrm{~h}$ à $16 \mathrm{~h}$

Pr. LERICHE

Transsexualisme

et Dr. WEBER

$16 \mathrm{~h}$ à $18 \mathrm{~h}$

Pr. COSNIER

Sexualité et phylogénèse

Mardi 9 Juin

$9 \mathrm{~h}$ à $10 \mathrm{~h}$

Dr. CHEVRET

Evénements de vie et sexualité

$10 \mathrm{~h}$ à $12 \mathrm{~h}$

Pr. ELCHARDUS

L'identité sexuelle

$14 \mathrm{~h}$ à $15 \mathrm{~h}$

Dr. BLACHERE

Les troubles de l'orientation sexuelle

$15 \mathrm{~h}$ à $17 \mathrm{~h}$

Dr. LHAMOTTE

$17 \mathrm{~h}$ à $18 \mathrm{~h}$

Dr. SALTEL

Délinquance sexuelle. Législation

Cancer et sexualité

Mercredi 10 Juin

$9 \mathrm{~h}$ à $11 \mathrm{~h}$

Dr. RIBES

Psychopathologie du couple

$11 \mathrm{~h}$ à $12 \mathrm{~h}$

Dr. RIBES

Hypnose et relaxation dans les difficultés sexuelles

$14 \mathrm{~h}$ à $16 \mathrm{~h}$

Pr. CZYBA

Stérilité et Sexualité. Sexualité et AMP

$16 \mathrm{~h}$ à $18 \mathrm{~h}$

Dr. CHARVIER

Handicap et sexualité

Jeudi 11 Juin

$9 \mathrm{~h}$ à $12 \mathrm{~h}$

Dr. CHEVRET

Psychothérapie du couple

$14 \mathrm{~h}$ à $16 \mathrm{~h}$

Dr. BONDIL

Traitement chirurgical de l'impuissance

$16 \mathrm{~h}$ à $18 \mathrm{~h}$

Dr. BLACHERE

Ethique médicale et thérapies sexuelles

Vendredi 12 Juin

$9 \mathrm{~h}$ à $12 \mathrm{~h}$

Dr.GIULIANO

Traitements médicaux de l'impuissance et injections intra caverneuses

14h à 16h Mme Mc DOUGALL La puissance masculine : approche psychanalytique. 